\title{
Circumstellar Carbonaceous Material Associated with Late-Type Dusty WC Wolf-Rayet Stars
}

\author{
Jean E. Chiar \\ NASA-Ames Research Center, Mail Stop 245-3, Moffett Field, CA \\ 94035, USA and SETI Institute
}

A.G.G.M. Tielens \& E. Peeters

Kapteyn Institute/SRON, Groningen, $N L$

\begin{abstract}
Our own solar system and other planetary systems are composed of organic dust created in the outflows in dying stars. Here, we examine an unlikely source of carbonaceous material in interstellar space, the harsh environment of Wolf-Rayet (WR) stars. We have used spectroscopic data from the Short Wavelength Spectrometer on the Infrared Space Observatory to examine infrared emission and absorption features in the 6 to $9 \mu \mathrm{m}$ spectral region of hot massive WC-type WR stars. We conclude that these features are due to amorphous carbon dust in their circumstellar environment.
\end{abstract}

\section{Introduction}

Infrared (IR) spectroscopy is a powerful tool being applied to study the evolutionary link between interstellar and circumstellar carbon-rich dust and the planetary systems that form from these materials. Carbonaceous dust composition is likely to be the result of variations in chemical and physical conditions and history in different astrophysical environments.

Despite their harsh environment - fast stellar winds and high stellar effective temperatures - some WC-type Population I ${ }^{1}$ Wolf-Rayet (WR) stars are known to produce copious amounts of carbon dust in their winds. WR stars represent one of the final stages of stellar evolution for massive stars. Late-type (i.e., WC9, WC10) WC stars are now known to be surrounded by heated circumstellar dust (Williams et al. 1987), although the process by which this dust forms and survives in the harsh WC-star environment is not well-understood (Cherchneff et al. 2000). The dust-producing WC stars have been divided into subtypes depending on the regularity of their dust formation. Of the five WC stars with the densest dust shells studied by van der Hucht et al. (1996), three (WR 104, WR 112, WR 118) are classified as persistent dust makers, one (WR 98a) is classified as having variable dust formation, and one (WR 48a) exhibits episodic

\footnotetext{
${ }^{1}$ Population I stars are relatively young stars, less than a few billion years old. They are metalrich, meaning that they are rich in elements heavier than hydrogen and helium.
} 


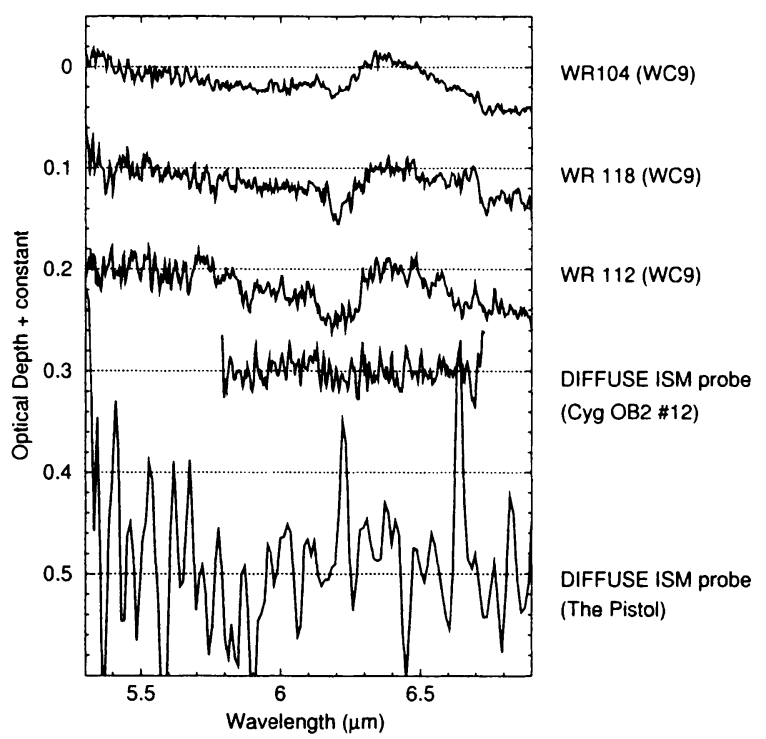

Figure 1. The $6.2 \mu \mathrm{m}$ absorption feature in the spectra of the WCtype WR stars. This absorption feature is missing in the diffuse ISM probe spectra which are obscured by similar amounts of interstellar dust. Adapted from Chiar \& Tielens 2001.

dust formation (Williams 1999). In general, dust formation appears to be the effect of colliding winds in WC+OB binary systems (e.g., Williams 1997; van der Hucht 2001 and references therein).

We have recently analyzed spectroscopic data from the Short Wavelength Spectrometer (SWS) onboard the Infrared Space Observatory (ISO). In this paper we present a summary of absorption and emission in the 6 to $9 \mu \mathrm{m}$ region, due to carbonaceous material in the circumstellar environment of some dusty WC-type WR stars (Chiar \& Tielens 2001; Chiar, Peeters, \& Tielens 2002).

\section{Absorption at 6.2 $\mu \mathrm{m}$ toward WR stars with Dense Dust Shells}

Previous analyses of the 5 to $8 \mu \mathrm{m}$ spectra of dusty WC-type stars attributed an absorption feature at $6.2 \mu \mathrm{m}$ to the $\mathrm{C}-\mathrm{C}$ stretching vibration in polycyclic aromatic hydrocarbons (PAHs) in the general diffuse interstellar medium (ISM) along the line of sight. However, we showed that this absorption feature is not detected in lines of sight which sample only diffuse ISM dust (Chiar \& Tielens 2001). Figure 1 shows the optical depth $\left(=-\ln \left[\mathrm{flux}_{\text {spectrum }} /\right.\right.$ flux $\left.\left._{\text {continuum }}\right]\right)$ spectra of the WC-type WR stars WR 104, WR 118, WR 112; and Cygnus OB2 \#12 and the "Pistol star." The latter two objects probe diffuse ISM dust and are obscured by 10 and 29 magnitudes of visual extinction, respectively. For the WC stars shown, the average total visual extinction is $\sim 10$ magnitudes; most of this extinction is due to interstellar dust, about 1 magnitude of this is thought to be due to circumstellar dust. Despite the similar amount of total visual extinction toward the WC-type stars and the diffuse ISM probes, only the WC-type stars 
exhibit absorption at $6.2 \mu \mathrm{m}$. Therefore, the absorption cannot be the result of dust in the diffuse ISM. We attribute the $6.2 \mu \mathrm{m}$ feature to the C-C stretch of aromatic carbonaceous material in the circumstellar environment of the WC stars. Based on the integrated absorption, the intrinsic strength of amorphous carbon and the small ( $\sim 1$ magnitude) visual extinction, large $(\sim 1 \mu \mathrm{m})$ dust grains are inferred. In spite of the difficulty of condensing large grains in the harsh WC-star environment, subsequent studies (of WR 112 and WR 118) also suggest the presence of large grains in the dust shells of these stars (Marchenko et al. 2002; Yudin et al. 2001).

\section{Unidentified Infrared (UIR) Emission bands of Wolf-Rayet 48a}

The IR spectrum of the WC-type star WR 48a exhibits a weak, asymmetric emission band at $\sim 6.4 \mu \mathrm{m}$, and a symmetric emission band at $7.9 \mu \mathrm{m}$ (Fig. 2) (Chiar, Peeters, \& Tielens 2002). These positions are close to the characteristic wavelengths of the UIR emission bands, generally ascribed to PAHs, near 6.2 and $7.7 \mu \mathrm{m}$. Due to the hydrogen deficiency of the circumstellar dust around WR $48 \mathrm{a}$, we attribute these features to amorphous carbon, rather than PAHs.

The $6.4 \mu \mathrm{m}$ feature in the WR $48 \mathrm{a}$ spectrum resembles that seen by Harrington et al. (1998) toward the hydrogen-deficient planetary nebulae (PNe) Abell 78 and IRAS 15154-5258. However, only one of the later sources shows a hint of broad emission near $8 \mu \mathrm{m}$. The observed $7.9 \mu \mathrm{m}$ feature toward WR $48 \mathrm{a}$ instead resembles those of the Population $\mathrm{II}^{2}$ PNe with WC10 central stars, Hen 2-113 and IRAS 07027-7934, and of the isolated Herbig AeBe star (an intermediate mass pre-main sequence star) HD 100546 (see Fig. 2 and Fig. 15 in Peeters et al. 2002). Unlike WR $48 \mathrm{a}$, these three sources show a " 6.2 " $\mu \mathrm{m}$ feature which peaks shortward of $6.3 \mu \mathrm{m}$.

Physical conditions are known to have an effect on the profiles (width, shape, central wavelength) of the UIR bands (Peeters et al. 2002). The carbon-rich and hydrogen-deficient conditions in the objects discussed above (with the exception of HD 100546) have the effect of shifting the "6.2" and "7.7" $\mu \mathrm{m}$ features toward longer wavelengths. An additional requirement may be associated with shockinduced dust condensation or modification of the PAHs. However, shocks are probably not responsible for the shift of the "6.2" $\mu \mathrm{m}$ feature toward HD 100546. For WR 48a, dust is formed in the interaction region of the winds of the O-star and the WR star (e.g., Usov 1991). In the [WC10]-PNe systems, interaction between the fast wind from the WC nucleus with the cooler slowly-moving ejecta of the pre-PN phase produces the necessary shock conditions.

\section{Future Directions}

It is possible that WR 48a provides a snapshot of the general dust condensation process in WC-type stars, frozen out due to the transient nature of the dust formation process in this object; a stage which is obfuscated in other dusty WC stars due to rapid dust growth. Additional observations with future space and

\footnotetext{
${ }^{2}$ Population II stars are relatively "old" stars - 2 to 4 billion years old - that are metal-poor.
} 

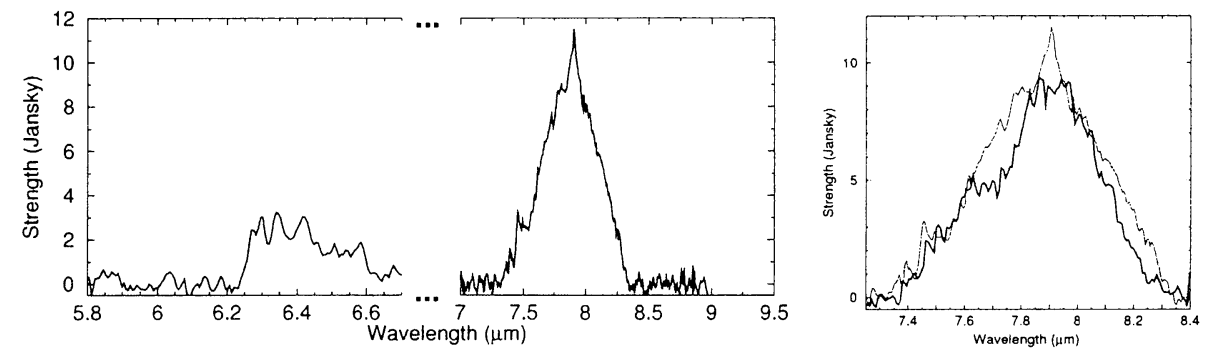

Figure 2. [Left] Mid-infrared spectra of WR 48a showing emission features at $\sim 6.4$ and $7.9 \mu \mathrm{m}$. [Right] The $7.9 \mu \mathrm{m}$ feature for WR $48 \mathrm{a}$ (thin line) compared with that of the PN-[WC10] object, Hen 2-113 (thick line). Adapted from Chiar, Peeters, \& Tielens 2002.

airborne missions will help us study the relationship between the WC star evolutionary status (spectral type), the dust shell characteristics, and the appearance of absorption/emission due to carbonaceous material.

Acknowledgments. J.E.C. is supported by NASA's Long Term Space Astrophysics Program under grant 399-20-61-02.

\section{References}

Cherchneff, I., Le Teuff, Y., Williams, P., \& Tielens, A. 2000, A\&A, 357, 572

Chiar, J. E., Peeters, E., \& Tielens, A. G. G. M. 2002, ApJ, 579, L91

Chiar, J. E., \& Tielens, A. G. G. M. 2001, ApJ, 550, L207

Harrington, J. P., Lame, N. J., Borkowski, K. J., Bregman, J. D., \& Tsvetanov, Z. I. 1998, ApJ, 501, L123

Marchenko, S. V., Moffat, A. F. J., Vacca, W. D., Côté, S., \& Doyon, R. 2002, ApJ, 565, L59

Peeters, E., Hony, S., Van Kerckhoven, C., Tielens, A. G. G. M., Allamandola, L. J., Hudgins, D. M., \& Bauschlicher, C. W. 2002, A\&A, 390, 1089

Usov, V. V. 1991, MNRAS, 252, 49

van der Hucht, K. A. 2001, New Astronomy Review, 45, 135

van der Hucht, K. A., et al. 1996, A\&A, 315, L193

Williams, P., van der Hucht, K., \& Thé, P. 1987, A\&A, 182, 91

Williams, P. M. 1997, Ap\&SS, 251, 321

Williams, P. M. 1999, in IAU Symp. 193: Wolf-Rayet Phenomena in Massive Stars and Starburst Galaxies, Vol. 193, 267

Yudin, B., Balega, Y., Blöcker, T., Hofmann, K.-H., Schertl, D., \& Weigelt, G. 2001, A\&A, 379, 229 